\title{
Multiscale Simulation of Gas-Liquid Flows with Droplets
}

\author{
Jimin Wang*, Feng Zhu, Xiaofang Liu and Wenke Li \\ School of energy and environment, Anhui University of Technology, Maan'shan 243002, Anhui, China \\ ${ }^{*}$ Corresponding author
}

\begin{abstract}
Multiscale phenomena represent a common challenge in many areas, including chemical engineering. Multiscale problems in gas-liquid flows, accompanied with highly turbulent flow and complex geometry of internals, make it difficult to achieve the fast and accurate simulation. In this report, special emphasis is focused on multiscale modeling strategies of multiphase flow problems of practical interest liking gas-liquid flows with droplets. Sophisticated interface-tracking algorithms such as Volume-of-Fluid, Level-set, and Front-tracking methods are briefly overviewed. Multiscale simulation of a variety of industrial applications such as atomization and sprays is subsequently discussed. This presentation paved the way for multiscale simulation of gas-liquid flows in industrial processes to optimize the operating conditions in manufacturing, determine proper reactor design and scale-up, and correctly interpret data in research and pilot-plant work.
\end{abstract}

Keywords-multiscale simulation, gas-liquid flows, droplets, atomization, spray

\section{INTRODUCTION}

A large set of physical systems of industrial and scientific relevance involve interactions between phenomena occurring at various scales. Consequently, multiscale simulation has been recognized as a powerful tool to improve the performance of process equipment, in particular in providing physical insight into fundamental physics and aspects of the phenomenon that are difficult to be examined experimentally. However, multiscale simulations of a physical process involving temporal and spatial scales require huge computational resources and time. Therefore, hybrid multiscale methods are needed to resolve the physics approximately or thoroughly at different scales and interaction between them via an interface sharing information. Various multiscale bridging methods have been developed to resolve the multiscale problems in the thermal and fluid science, namely, the heterogeneous multiscale method, the variational multiscale method, the discontinuous Galerkin method and the equation-free method. Generally, for multiphase non-equilibrium system, multiscale simulations involving mesoscopic particles suspended in a macroscopic fluid have been usually performed using Euler-Euler and EulerLagrange models. In the Euler-Euler approach, the dispersed small-scale phase is collectively modeled using a concentration field. Statistical models are employed to simulate the cumulative effect of the dispersed phase on the suspension fluid. In contrast, Euler-Lagrange methods involve solving the continuous phase using numerical schemes such as Finite Differences, Finite Elements or Finite Volumes and performing discrete Lagrange advection of the dispersed phase. The hydrodynamic forces acting on the particles are incorporated using empirical correlations and theoretical models. The effect of the particles on the underlying fluid is modeled as a source term in the momentum equations. Indeed, the volume of literature on the subject of multiscale of gas-liquid flows is so huge that summarising every study would appear to be beyond the scope of any single up to-date review. A summary of research work on the important and specific applications of atomization and spray has been considered as the appropriate subject of the current review. Thus, it is the aim of the contribution to present an overview of multiscale strategies of gas-liquid flows with droplets. Various multiscale bridging methods are introduced. The sophisticated numerical schemes have been developed to capture or track the interface evolution, such as the Volume-of-Fluid (VOF), Level-Set (LS), and Front-Tracking (FT) methods. Multiscale simulation of a variety of industrial applications such as atomization and sprays is subsequently discussed. This review aims to provide a comprehensive and understandable account of the theoretical foundation of the approach for multiscale gas-liquid flows, and attempts to summarize these advances and also outline opportunities for further development of the multiscale approach. Meanwhile, a pathway to comprehensive multiscale modeling is still to be found.

\section{Multiscale Methods}

\section{A. Heterogeneous Multiscale Method}

The heterogeneous multiscale method relies on an efficient coupling between the macroscopic and microscopic models [1]. In case the macroscopic model is not explicitly available or is invalid in part of the domain, the microscopic model is used to supply the necessary data for the macroscopic model. Scale separation is exploited so that coarse-grained variables can be evolved on macroscopic spatial/temporal scales using data that are predicted based on the simulation of the microscopic process on microscale spatial/temporal domains. The compression operators are in general local/ensemble averages, projection to low order moments, or slow manifolds. The reconstruction operator does the opposite. There are two main components in the heterogeneous multiscale method: An overall macroscopic scheme such as finite element method, discontinuous Galerkin method, and Runge-Kutta method for a state variable, and estimating the missing macroscopic data from the microscopic model by coupling principles as found in the short monograph. 


\section{B. Variational Multiscale Method}

The variational multiscale method (VMS) was originally developed for enriching continuum solutions with fine scale continuum description, which deal with different and new variant of mixed variational formulations when additional terms are inserted in the Navier-Stokes equations or when different physics is applied [2]. The velocity and the pressure unknowns can be split into two components corresponding to different scales or levels of resolution: resolvable coarse-scale and unresolved fine-scale. The unresolved fine-scales are then modeled using residual based terms that are derived consistently. The static condensation consists of substituting the fine-scale solution into the large-scale problem providing additional terms, tuned by a local stabilizing parameter. The latter enhances the stability and accuracy of the standard Galerkin formulation. Thus, the mixed-finite element approximation of the time-dependent incompressible problem with surface tension can be obtained.

\section{Discontinuous Galerkin Method}

The discontinuous Galerkin (DG) methods are locally conservative, stable, and high-order accurate methods which can easily handle complex geometries, irregular meshes with hanging nodes, and approximations that have polynomials of different degrees in different elements [3]. DG methods combine features of the finite element and the finite volume framework. Unlike traditional continuous Galerkin methods that are conforming, the DG method works over a trial space of functions that are only piecewise continuous, and thus often comprise more inclusive function spaces than the finitedimensional inner product subspaces utilized in conforming methods. The main components of the method are discontinuous approximations, element-by-element Galerkin weak formulations and the numerical traces.

\section{Equation-Free Method}

Equation-free modeling is designed for a class of complicated systems in which one observes evolution at a macroscopic, coarse scale of interest, while accurate models are only given at a finely detailed, microscopic, level of description [4]. The framework empowers one to perform macroscopic computational tasks over large space-time scales using only appropriately initialized microscopic simulation on short time and small length scales. The methodology eliminates the derivation of explicit macroscopic evolution equations when these equations conceptually exist but are not available in closed form. A key tool is the coarse time-stepper, which implements a time step of an unavailable macroscopic model as a three-step procedure: lifting, simulation and restriction.

\section{Multiphase Flows Model}

\section{A. Euler-Euler Model}

Here, each fluid is treated as a continuous phase by an Euler approach. The equations of motion for phase $k$ in an Euler-Euler simulation are generally given as follows:

$$
\frac{\partial \rho^{k} \alpha^{k}}{\partial t}+\nabla \times \alpha^{k} \rho^{k} \mathrm{u}^{k}=0
$$

$$
\frac{\partial \rho^{k} \alpha^{k} \mathrm{u}^{k}}{\partial t}+\nabla \times \alpha^{k} \rho^{k} \mathrm{u}^{k} \mathrm{u}^{k}=-\alpha^{k} \nabla P+\alpha^{k} \rho^{K} g-\nabla \times \alpha^{k} \tau^{k}+F^{k}
$$

where $\alpha, \rho, t, u$, and $\tau$ is the volume fraction, density, time, velocity vector, and shear stress; $P$ is the pressure. $g$ is the gravity acceleration vector. $F_{k}$ represents the interfacial forces between the two phases. The superscript $k=l$ or $g$ denotes the liquid or gas phase.

\section{B. Euler-Lagrange Model}

The Euler-Lagrange approach is used to denote a family of modeling and simulation techniques wherein droplets or particles are represented in a Lagrange reference frame while the carrier phase flow field is represented in an Euler frame. The governing equations for the continuous gas phase include continuity, momentum, energy, and species conservations. The effect of turbulence is typically encountered by means of the k$\varepsilon$ model. The dispersed phase trajectories are calculated using a Discrete Phase Model (DPM) formulated in Lagrange moving reference frame.

$$
\begin{gathered}
\frac{d x_{p}}{d t}=v_{p} \\
m_{p} \frac{d v_{p}}{d t}=F_{p, \text { flow }}+F_{p, \text { external }}+F_{p, \text { collisions }}
\end{gathered}
$$

where $m_{p}, x_{p}$, and $v_{p}$ are the mass, position, and velocity, respectively, of the pth particle; and $F_{p, f l o w}, F_{p \text {,external, and }}$ $F_{p \text {,collisions }}$ are the forces acting on the $p t h$ particle generated by the flow, external force, and force caused by collisions between the particles, respectively. Among the forces acting on the Lagrange particles, the drag force generated by the background fluid plays an important role.

\section{INTERFACE-TRACKING ALGORITHMS}

\section{A. Volume of Fluid}

In the VOF method, a volume fraction $\mathrm{f}$ is defined in each computational cell as the fraction of the cell containing liquid. If the cell is completely filled with liquid then $f=1$ and if it is filled with gas then its value should be 0 . At the gas-liquid interface the value of $f$ is between 0 and 1 . The motion of the interface is captured through the evolution of the volume fraction that takes the form [5]:

$$
\frac{\partial f}{\partial t}+\nabla \cdot f \mathbf{u}+\nabla \cdot\left(f(1-f) \mathbf{u}_{r e l}\right)=0
$$


The third term on the left hand side of the above equation is an artificial compression term which is active only in the interface region. $\boldsymbol{U}_{r e l}$ is a velocity field suitable to compress the interface.

\section{B. Front-Tracking Method}

Front-tracking modeling marks the interface but introduce an additional computational element, the interface grid, that explicitly marks the position of the interface, only modified near the front to make a grid line follow the interface, and is used for the fluid within each phase. An indicator function, $I$, that is 1 where one particular fluid is and 0 elsewhere. The interface itself is marked by a nonzero value of the gradient of the indicator function. If the density of each phase is assumed to be constant, it can be written in terms of the constant densities and the indicator function at each grid point by [6-7]

$$
\rho=\rho_{0}+\left(\rho_{l}-\rho_{0}\right) I
$$

Here, $\rho_{l}$ is the density where $I=1$ and $\rho_{0}$ is the density where $I=0$. The material properties are, in general, discontinuous across the interface, and are represented by $\delta$ functions to add the appropriate interface terms to the governing equations.

\section{Level-Set Method}

The level set method enables the localization of the interface between two phases. It is a signed distance function and it is a widely used tool in different fields such as crystal growth, image restoration or surface reconstruction. Let $\Omega$ be the whole domain, $\Omega_{l}$ the liquid domain and $\Omega_{\mathrm{g}}$ the gas domain. The level set function is a signed distance function from the interface $\Gamma=\Omega_{l} \cap \Omega_{g}$ defined at each node $X$ of $\Omega$ as follows:

$$
\alpha(X)=\left\{\begin{array}{c}
-\operatorname{dist}(X, \Gamma), X \in \Omega_{l} \\
0, X \in \Gamma \\
\operatorname{dist}(X, \Gamma), X \in \Omega_{g}
\end{array}\right.
$$

The evolution of the level set function is described by the following transport equation:

$$
\frac{d}{d t} \alpha(x, t)=\frac{\partial \alpha}{\partial t}+u \cdot \nabla \alpha=0
$$

The level set, as a distance function, verifies $\|\alpha\|=1$.

\section{Multiscale Simulation of Atomization AND SPRAY}

\section{A. Spray Drying}

Spray drying is an extensively used technology in process engineering for receiving small particles by rapid moisture evaporation [8-9]. The process involves multiphase transport phenomena between drying agent, droplets and particles, and chamber boundaries. These transport phenomena occur on multiple scales and include simultaneous external and internal heat and mass transfer in each phase. Nevertheless, the mechanisms that control spray formation and evolution are far from being understood. A liquid jet upon spray-drying breaks up into small droplets that are orders of magnitude smaller than its diameter. Direct numerical simulations of spray drying are exceedingly expensive computationally. Thus, an advanced spray-drying model of single droplet drying kinetics based on a multiscale multiphase approach is coupled to an EulerLagrange description of gas-spray mixing and the overall formulation is solved numerically using a CFD technique. Achievements and results of the comprehensive scientific research on multiscale multiphase modeling of transport phenomena in spray-drying processes are summarized [10-11]. This advancement provides engineers with more accurate, high-quality tools for innovative design of spray-dryer systems and for choosing suitable operating conditions, enabling engineering of particles with desired properties.

\section{B. Atomization}

Modeling and simulation of atomization is challenging due to the existence of a wide range of length scales. A multiscale simulation approach for atomization was proposed, where the large-scale interfaces are resolved by the VOF method and the small droplets by the Lagrange point-particle (LPP) model. To accurately compute the dynamics of the LPP droplets that are larger than the grid spacing, a new model of the momentum coupling and the two-way conversion between the LPP droplets and the resolved flow is proposed [12]. Moreover, collisions between two particles are efficiently predicted using a spatial-hashing algorithm. The atomization simulation presented in the current study has been performed for a high liquid-gas density ratio. The process reduces the computational cost considerably, enabling us to simulate a swarm of droplets spreading outward from the liquid jet [13].

\section{Liquid Stream}

For high-pressure water stream, fountain, waterfall, and smash waves, fluid details and their natural interaction with nearby liquids must be vividly simulated from apparent droplets to minute droplets and visible fog-like sprays. Despite the fact that Lagrange particles are widely employed for representing individual droplets and Euler grid-based method is ideal for volumetric spray modeling, using either one alone has encountered tremendous difficulties when effectively simulating droplet/spray mixture phenomena. Therefore, a novel particle-grid hybrid solution with Fluid Implicit Particle (FLIP) method was proposed to tackle multiscale fluid modeling as well as their interactive and realistic simulation. The hybrid model can fully leverage the respective advantages of Lagrange and Euler methods, wherein particles are employed for individual droplet representation and the gridbased density field is introduced for volumetric spray modeling [14]. At present, the hybrid model ignore the spray-droplet transition, so that it shall still continue to study the spray condensation mechanism and involve other environmental factors such as temperature, floating dust, etc., which could broaden the practical spectra of the method and its utility. 


\section{Spray Combustion}

In recent years, despite the significant progress made in automobile engines for energy conservation and environmental protection, our understanding of spray-combustion phenomena is far from complete. The current trend requires simultaneous consideration of the liquid phase, which may contain droplets and ligaments [15]. To simulate the behaviors of multiscale free surfaces of spray combustion, the liquid-film breakup was simulated by using a particle method, and the secondary-drop breakup was simulated by using a discrete droplet model (DDM). With these methods, motions of macro-free surfaces larger than the computational grid sizes were mainly simulated by cubic interpolated propagation (CIP), and motions of microfree surfaces smaller than the grid sizes were mainly simulated by the moving-particle semi-implicit (MPS) method [16].

\section{E. Spray Processing}

Metal-matrix-composite (MMC) particles for thermal spray coating applications may be produced through mixing of ceramic particles and metallic droplets. The complex interactions between metallic droplets and ceramic particulates in the dispersed multiphase flow during spray processing are quantitatively described based on multiscale modelling and simulation, which deal with the mixing process of metallic droplet sprays and ceramic particulate jets during melt atomization and spray, the collision process of ceramic particulates with an arbitrary metallic droplet, and the penetration process of a ceramic particle into a metallic droplet. It is shown that the characteristic collection efficiency increases with increasing atomization gas pressure [17]. However, more advanced solidification and rheological models are necessary in future to obtain more reliable results. The integral spray model can be also expanded to other spray applications, e.g., spray drying process and wet scrubbing system.

\section{CONCLUDING REMARKS}

Most phenomena and processes in science and engineering are multiscale in nature. With the rapid development in science and technology the importance of study from multiscale view point becomes more and more obvious. Understanding the multiscale complexity plays an important role in the study of structure-function relationships of materials, mixing and transfer in reactors as well as the system integration. During the last few years, studies of multiscale simulation published provide engineers with more accurate, high-quality tools for innovative design of multiscale systems and for choosing suitable operating conditions, enabling engineering of particles with desired properties. Particularly, a clearer picture and deeper understanding framework with mathematical model, numerical simulation, intelligent integration, comprehensive decision, and virtual engineering for multiscale spatio-temporal structures may be successfully developed to explore thermophysical mechanism and optimization strategy of process engineering.

However, the numerical study for the multiscale simulation of multiphase flow problems is still in its childhood and its application is in its infancy. Further, researches are highly required to establish robust and quick-convergent numerical solution approaches. The nonlinear and multiscale interactions and nonequilibrium effects in multiphase flow result in a rich variety of flow phenomena spanning many flow regimes. Therefore, multiscale modelling of multiphase flows are being developed, such as the coupled population balance-CFD simulations, are not only extremely useful for fundamental research, but also have the potential to complement traditional chemical engineering methods in an industrial context. In particularly, a novel multiscale modeling approach for multiphase flows in microtechnology and process intensification is developed within framework of the Lattice Boltzmann method. A broader application of the proposed multiscale modeling could enable efficient design optimization, as most of the computational effort would shift to the coarser scale. More sophisticated numerical integration schemes must be applied which are much more immune to numerical diffusion.

\section{ACKNOWLEDGMENT}

The authors gratefully acknowledge the support of the National Science Foundation of China (51306001 and 51576002), Natural Science Foundation of Anhui Province (1708085ME108).

\section{REFERENCES}

[1] E.Weinan, B.Engquist, and Z.Huang, "Heterogeneous multiscale method: A general methodology for multiscale modeling," Physical Review B, vol.67, pp.552-555, 2003.

[2] M.Khalloufi, Y.Mesri, R.Valette, E.Massoni, and E.Hachem, "High fidelity anisotropic adaptive variational multiscale method for multiphase flows with surface tension," Computer Methods in Applied Mechanics \& Engineering, vol. 307, pp.44-67, 2016.

[3] B.Cockburn., "Discontinuous Galerkin Methods," Journal of Applied Mathematics and Mechanics, vol.83, pp.731-754, 2003.

[4] I.G.Kevrekidis, and G.Samaey, "Equation-Free Multiscale Computation: Algorithms and Applications," Annual Review of Physical Chemistry, vol.60, pp.321-344, 2009.

[5] X.-G.Li, and U.Fritsching, "Numerical investigation of solid particle penetration into liquid droplet," Materialwissenschaft Und Werkstofftechnik, vol.45, pp.666-682, 2014.

[6] S.O.Unverdi, and G.Tryggvason, "A front-tracking method for viscous, incompressible, multi-fluid flows," Journal of Computational Physics, vol.100, pp.25-37, 1992.

[7] G.Tryggvason, B.Bunner, A.Esmaeeli, D.Juric, N.Al-Rawahi, and W.Tauber, et al., "A front-tracking method for the computations of multiphase flow," Journal of Computational Physics, vol.169, pp.708759, 2001

[8] N.Ashgriz, A. L.Yarin, A.L.Yarin, N.Ashgriz, X.Li, and A.Sarchami, et al., Handbook of Atomization and Sprays. Berlin, Germany: SpringerVerlag GmbH, 2011, pp.849-850

[9] X.Jiang,G.A.Siamas,K.Jagus, and T.G.Karayiannis, "Physical modelling and advanced simulations of gas-liquid two-phase jet flows in atomization and sprays,". Progress in Energy \& Combustion Science, vol.36, pp.131-167, 2010.

[10] A.Levy, "Multi-Scale Multiphase Modeling of Transport Phenomena in Spray Drying Processes," Drying Technology, vol.33, pp.2-23, 2015.

[11] I.Borde,"Three-Dimensional Spray-Drying Model Based on Comprehensive Formulation of Drying Kinetics," Drying Technology, vol.30, pp.1256-1273, 2012.

[12] Y.Ling, S.Zaleski, and R.Scardovelli, "Multiscale simulation of atomization with small droplets represented by a Lagrangian pointparticle model," International Journal of Multiphase Flow, vol.76, pp.122-143, 2015. 
[13] G.Tomar, D.Fuster, S.Zaleski, and S.Popinet, "Multiscale simulations of primary atomization," Computers \& Fluids, vol.39,pp.1864-1874, 2010.

[14] L.Yang, S.Li, A.Hao, and H.Qin, "Hybrid Particle - grid Modeling for Multi - scale Droplet/Spray Simulation," Computer Graphics Forum, vol.33, pp.199-208, 2014.

[15] A.L.Sánchez, J.Urzay, and A.Liñán, "The role of separation of scales in the description of spray combustion," Proceedings of the Combustion Institute, vol.35, pp.1549-1577, 2015.

[16] E.Ishii, M.Ishikawa,Y.Sukegawa, and H.Yamada, "Secondary-DropBreakup Simulation Integrated With Fuel-Breakup Simulation Near Injector Outlet," Journal of Fluids Engineering, vol.133,pp. 0813021-8, 2011.

[17] X.-G.Li, L.Heisterüber, L.Achelis, and U.Fritsching, "Multiscale descriptions of particle-droplet interactions in multiphase spray processing," International Journal of Multiphase Flow, vol.80, pp.15-28, 2016. 